\title{
Amenazas para la salud en el Río Sonora: análisis exploratorio de la calidad del agua reportada en la base de datos oficial de México
}

\author{
Health threats in the Sonora River: exploratory analysis of \\ water quality reported in the official database of Mexico
}

\author{
Rolando E. Díaz-Caravantes ${ }^{1}$, Héctor Duarte-Tagles², Francisco M. Durazo-Gálvez²
}

Forma de citar: Díaz-Caravantes RE, Duarte-Tagles H, Durazo-Gálvez FM. Amenazas para la salud en el Río Sonora: análisis exploratorio de la calidad del agua reportada en la base de datos oficial de México. Rev Univ Ind Santander Salud. 2016; 48(1): 91-96. DOI: http://dx.doi.org/10.18273/revsal.v48n1-2016010 (c) (1) ()

\section{RESUMEN}

El 6 de agosto de 2014 ocurrió en el Río Sonora, México, lo que fue llamado "el peor desastre ambiental de la industria minera del país" cuando la mina Buenavista del Cobre derramó alrededor de 40,000 metros cúbicos de lixiviado ácido. Con el fin de avanzar en el conocimiento de las consecuencias de este evento, se analizó la base de datos del monitoreo de la calidad del agua subterránea que aparece en el portal electrónico del Fideicomiso Río Sonora. Por las implicaciones que este evento tiene para la salud, los valores reportados se confrontaron con los límites máximos permisibles establecidos en la Norma Oficial Mexicana vigente. Asímismo, para enriquecer la discusión, se analizó el caso del arsénico, comparando los valores reportados en relación con la Norma Mexicana y con la Guía de calidad para el agua de la Organización Mundial de la Salud.

Palabras clave: Calidad del agua, Río Sonora, Buenavista del Cobre, vertimiento.

\begin{abstract}
On August 6th, 2014, the event labelled as the "worst environmental disaster of the mining industry in Mexico" ocurred in the Sonora River, when Buenavista del Cobre mine spilled around 40,000 cubic meters of an acidic leachate. In order to expand our knowledge of the consequences of this event, the water quality monitoring data base was analyzed for groundwater from the Río Sonora trust fund webpage. Due to the health relevance of the spill, the reported data was compared to the established maximum allowance values of Mexican regulations. Furthermore, for an enhanced discussion, the case of arsenic was also analyzed, comparing the data with Mexican regulations as well as with the WHO guidelines for drinking water.
\end{abstract}

Keywords: water quality, Río Sonora, Buenavista del Cobre, overspill.

1. Colegio de Sonora

2. Universidad de Sonora

Correspondencia: Rolando E. Díaz-Caravantes. Dirección: Colegio de Sonora. Avenida Obregón \#54, Colonia Centro, Hermosillo, Sonora. Teléfono: 52 (662) 259530. Correo electrónico: rdiaz@colson.edu.mx 


\section{INTRODUCCIÓN}

Las consecuencias de la actividad minera para el ambiente y la salud han sido documentadas en diversos estudios, sobre todo cuando ocurren accidentes que provocan que los seres humanos y otros organismos vivos queden expuestos a una gran cantidad de sustancias tóxicas ${ }^{1,2}$.

El 6 de agosto de 2014, la mina Buenavista del Cobre derramó alrededor de 40,000 metros cúbicos de lixiviado ácido a uno de los arroyos que alimenta al Río Bacanuchi, afluente a su vez del Río Sonora ${ }^{3}$. La cuenca del Río Sonora se localiza en el noroestecentro del estado de Sonora, México, y abarca un área total de 30,913 kilómetros cuadrados. El Río Sonora tiene una longitud de 294 kilómetros y fluye hacia el sur a la presa Abelardo L. Rodríguez, la cual tiene una capacidad de 219.5 millones de metros cúbicos y es contigua a la ciudad de Hermosillo, capital del estado de Sonora.

Las repercusiones para el ambiente y la salud de esta contingencia aún están en proceso de estimarse, pero el alto contenido de metales y metaloides disueltos en la solución acidulada vertida al cuerpo de agua -muchos de ellos considerados altamente tóxicos y cancerígenos- motivaron al entonces titular de la Secretaría de Medio Ambiente y Recursos Naturales (SEMARNAT) declararla como "el peor desastre ambiental de la industria minera del país"4. A raíz de este evento, el 15 de septiembre de 2014 se conformó un fideicomiso cuya finalidad es atender las consecuencias del derrame.

El Comité Técnico del fideicomiso está constituido por cinco miembros y es presidido por la SEMARNAT, la cual creó un portal electrónico accesible a través de internet, donde se presenta la información oficial sobre las acciones que se han implementado para atender el problema de la contaminación del Río Sonora derivado del derrame ${ }^{5}$.

El presente estudio es un reporte de caso cuya finalidad es mostrar algunos de los primeros hallazgos de un análisis de los valores de los metales y otros parámetros fisicoquímicos, que componen la base de datos del monitoreo del agua reportados por el Fideicomiso Río Sonora en su página de internet. Es importante aclarar que dicha base de datos se ha convertido en el portal oficial de las autoridades para informar al público del cumplimiento de las metas establecidas.

\section{MATERIALES Y MÉTODOS}

Se analizó la base de datos del monitoreo de la calidad del agua subterránea que aparece en el portal electrónico del Fideicomiso Río Sonora, con el fin de determinar la frecuencia de muestreo de cada uno de los 19 parámetros reportados para los 34 sitios de muestreo durante el período del 18 de agosto de 2014, al 24 de septiembre de 2015. Asimismo, los valores reportados se confrontaron con los límites permisibles establecidos en la Norma Oficial Mexicana vigente NOM-127SSA1-1994 "Salud ambiental. Agua para uso y consumo humano. Límites permisibles de calidad y tratamientos a que debe someterse el agua para su potabilización" así como el límite máximo recomendado de arsénico establecido en las Guías de Calidad para el Agua de Consumo de la Organización Mundial de la Salud (OMS) en su cuarta edición?

\section{RESULTADOS Y DISCUSIÓN}

En la Tabla 1 se presentan los 19 parámetros medidos y mostrados en el portal electrónico del fideicomiso para el agua subterránea. Como se observa, de los primeros 13 parámetros se tomaron más de mil muestras durante el período examinado; mientras que de los últimos seis se hicieron menos de 450 mediciones. En los registros se observó una cantidad considerable de casos en los que aparecía la etiqueta "ND" y otro tanto con la etiqueta "NE", de tal forma que del total de registros sólo una parte cuenta con un valor numérico, que en algunos casos llega a ser cerca de la mitad.

La base de datos no especifica el significado de la etiqueta "ND"; suponemos que puede tratarse de no detectable o no detectado, si esto es correcto, significa que en estos casos el instrumento utilizado para examinar la muestra no tuvo la sensibilidad suficiente para detectar un valor mínimo. Por otra parte, tampoco se especifica el significado de "NE"; suponemos que puede tratarse de no examinado, no estudiado o no especificado.

De los parámetros medidos, no todos cuentan con un límite permisible en la NOM 127-SSA1-1994, estos fueron etiquetados como NA (No Aplica) en la Tabla 1. De los 14 parámetros que sí están considerados en la norma mexicana, siete elementos tuvieron dos o menos casos dentro del límite permisible. Otros siete parámetros obtuvieron nueve o más casos fuera del límite. Estos últimos se muestran en la Tabla 2, y están ordenados quincenalmente según la fecha de muestreo. 
Amenazas para la salud en el Río Sonora: análisis exploratorio de la calidad del agua reportada en la base de datos oficial de México

Tabla 1. Total de registros según el parámetro medido y el límite permisible de la NOM 127-SSA1-1994.

\begin{tabular}{|c|c|c|c|c|c|c|c|c|}
\hline $\begin{array}{l}\text { Parámetro } \\
\text { medido }\end{array}$ & $\begin{array}{c}\text { Límite } \\
\text { permisible }\end{array}$ & Unidad & $\begin{array}{c}\text { Total de } \\
\text { Registros }\end{array}$ & $\begin{array}{l}\text { Registros } \\
\text { con valor } \\
\text { numérico }\end{array}$ & $\begin{array}{c}\text { Registros } \\
\text { fuera del } \\
\text { límite }\end{array}$ & $\begin{array}{c}\text { Registros } \\
\text { dentro del } \\
\text { límite }\end{array}$ & $\begin{array}{l}\text { Registros } \\
\text { "ND" }\end{array}$ & $\begin{array}{l}\text { Registros } \\
\text { "NE" }\end{array}$ \\
\hline Aluminio & 0,2 & $\mathrm{mg} / \mathrm{L}$ & 1180 & 867 & 102 & 765 & 209 & 104 \\
\hline Antimonio & NA & $\mathrm{mg} / \mathrm{L}$ & 1154 & 627 & NA & NA & 423 & 104 \\
\hline Arsénico & 0,025 & $\mathrm{mg} / \mathrm{L}$ & 1154 & 799 & 9 & 790 & 251 & 104 \\
\hline Bario & 0,7 & $\mathrm{mg} / \mathrm{L}$ & 1183 & 1066 & 0 & 1066 & 13 & 104 \\
\hline Cadmio & 0,005 & $\mathrm{mg} / \mathrm{L}$ & 1183 & 594 & 1 & 593 & 485 & 104 \\
\hline Cobre & 2 & $\mathrm{mg} / \mathrm{L}$ & 1183 & 761 & 0 & 761 & 318 & 104 \\
\hline Cromo & 0,05 & $\mathrm{mg} / \mathrm{L}$ & 1183 & 690 & 0 & 690 & 389 & 104 \\
\hline Fierro & 0,3 & $\mathrm{mg} / \mathrm{L}$ & 1183 & 1052 & 171 & 881 & 27 & 104 \\
\hline Manganeso & 0,15 & $\mathrm{mg} / \mathrm{L}$ & 1171 & 863 & 17 & 846 & 215 & 93 \\
\hline Mercurio & 0,001 & $\mathrm{mg} / \mathrm{L}$ & 1183 & 727 & 15 & 712 & 354 & 102 \\
\hline Níquel & NA & $\mathrm{mg} / \mathrm{L}$ & 1183 & 631 & NA & NA & 449 & 103 \\
\hline Plomo & 0,01 & $\mathrm{mg} / \mathrm{L}$ & 1183 & 585 & 2 & 583 & 495 & 103 \\
\hline Zinc & 5 & $\mathrm{mg} / \mathrm{L}$ & 1180 & 1041 & 0 & 1041 & 35 & 104 \\
\hline Sulfatos & 400 & $\mathrm{mg} / \mathrm{L}$ & 442 & 334 & 39 & 295 & 4 & 104 \\
\hline Turbiedad & 5 & UTN & 443 & 251 & 10 & 241 & 88 & 104 \\
\hline $\begin{array}{l}\text { Sólidos disueltos } \\
\text { totales }\end{array}$ & NA & $\mathrm{mg} / \mathrm{L}$ & 316 & 2 & NA & NA & 210 & 104 \\
\hline $\begin{array}{l}\text { Conductividad } \\
\text { electrolítica }\end{array}$ & NA & $\mu \mathrm{S} / \mathrm{cm}$ & 316 & 212 & NA & NA & 0 & 104 \\
\hline $\mathrm{pH}$ & $6.5-8.5$ & $\mathrm{UpH}$ & 316 & 212 & 0 & 212 & 0 & 104 \\
\hline Temperatura & NA & ${ }^{\circ} \mathrm{C}$ & 311 & 212 & NA & NA & 0 & 99 \\
\hline
\end{tabular}

Nota: $\mathrm{mg} / \mathrm{L}=$ miligramos por litro, $\mathrm{UTN}=$ unidades de turbiedad nefelométricas, $\mu \mathrm{S} / \mathrm{cm}=$ microsiemens por centímetro, $\mathrm{UpH}=$ unidades de $\mathrm{pH},{ }^{\circ} \mathrm{C}=$ grados Celsius.

Fuente: Elaboración propia con base en página oficial del Fideicomiso Río Sonora ${ }^{3}$.

Tabla 2. Registros fuera del límite permisible de la NOM 127-SSA1-1994 según la fecha de muestreo.

\begin{tabular}{|c|c|c|c|c|c|c|c|}
\hline Fechas de muestreo & Aluminio & Arsénico & Fierro & Manganeso & Mercurio & Sulfatos & Turbiedad \\
\hline 18-31 agosto 2014 & 29 & 0 & 50 & 1 & 3 & Sin muestreo & Sin muestreo \\
\hline 1-15 septiembre 2014 & 40 & 0 & 63 & 6 & 1 & Sin muestreo & Sin muestreo \\
\hline 16-30 septiembre 2014 & 27 & 5 & 26 & 0 & 9 & 2 & 1 \\
\hline 8 y 14 octubre 2014 & 2 & 1 & 8 & 2 & 2 & 6 & 3 \\
\hline 21 y 28 octubre 2014 & 4 & 3 & 12 & 1 & 0 & 3 & 2 \\
\hline 11 noviembre 2014 & 0 & 0 & 4 & 0 & 0 & Sin muestreo & Sin muestreo \\
\hline 9 diciembre 2014 & 0 & 0 & 6 & 0 & 0 & 4 & 3 \\
\hline 3 y 10 julio 2015 & 0 & 0 & 0 & 1 & 0 & 1 & 0 \\
\hline 17,24 y 31 julio 2015 & 0 & 0 & 2 & 2 & 0 & 7 & 1 \\
\hline 6 y 12 agosto 2015 & 0 & 0 & 0 & 1 & 0 & 7 & 0 \\
\hline 20 y 28 agosto 2015 & 0 & 0 & 0 & 2 & 0 & 7 & 0 \\
\hline 24 septiembre 2015 & 0 & 0 & 0 & 1 & 0 & 2 & 0 \\
\hline Total & 102 & 9 & 171 & 17 & 15 & 39 & 10 \\
\hline
\end{tabular}

Fuente: Elaboración propia con base en página oficial del Fideicomiso Río Sonora ${ }^{3}$. 
De la Tabla 2, resalta el hecho de que las mediciones fueron realizadas mayormente en los meses de agosto y septiembre de 2014, en los que hubo muestreo prácticamente todos los días. En el mes de octubre, los muestreos fueron semanales y ya para noviembre y diciembre sólo se realizó uno por mes. En la base de datos no aparecen muestreos entre enero y junio de 2015. En los meses de julio y agosto de 2015 se efectuaron muestreos semanales. En septiembre de 2015 aparecía un solo muestreo el día 24.

También destaca el dato de que la mayor parte de los casos fuera del límite permisible de la norma mexicana ocurrieron desde agosto hasta octubre de 2014; sin embargo, esto no fue así en todos los parámetros: para el manganeso, en 2015, fueron registrados siete de los 17 casos fuera del límite de la norma y para sulfatos fueron registrados 24 del total de los 39 .
Con el fin de mostrar las discrepancias entre los límites permisibles establecidos en la normatividad mexicana y los valores recomendados en las guías de la OMS, se analizó el caso del arsénico, ya que existe evidencia del efecto tóxico del metaloide por exposición crónica a concentraciones mayores a 0.01 miligramos por litro $(\mathrm{mg} / \mathrm{L})$ en agua para consumo humano ${ }^{8,9}$.

Como se observa en las tablas anteriores, si tomamos el límite permisible establecido en la NOM 127-SSA11994 para el arsénico de $0.025 \mathrm{mg} / \mathrm{L}$, solamente nueve muestras lo excedían. En cambio, si tomamos la guía de la OMS para el arsénico de $0.01 \mathrm{mg} / \mathrm{L}$, resulta que son 120 casos los que estuvieron fuera de este límite máximo. La distribución temporal tanto de los casos que excedían la norma como de los casos que rebasaban la guía de la OMS se observa en la Figura 1.

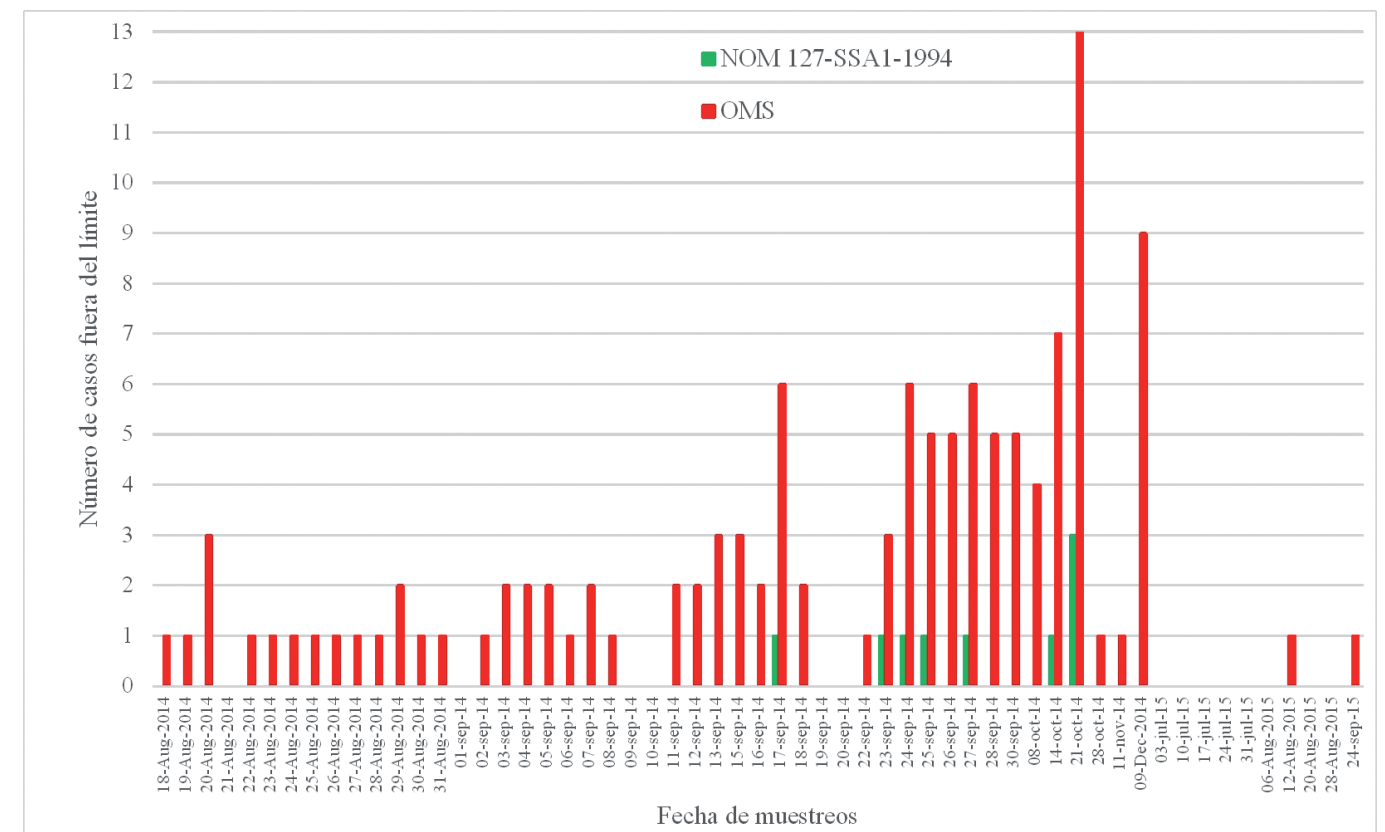

Figura 1. Número de registros de arsénico fuera del límite de la NOM 127-SSA1-1994 y de la OMS según las fechas de muestreo

Como se aprecia en la Figura 1, de acuerdo con la Norma, los límites fueron excedidos entre el 17 de septiembre y el 21 de octubre de 2014. Si se utiliza la guía de la OMS se tiene un panorama diferente, pues desde la primera fecha de muestreo ya existía un caso. De forma similar a la norma, los muestreos con más casos comenzaron el 17 de septiembre, pero a diferencia de ésta, todavía para el 9 de diciembre de 2014 existían nueve casos; no sabemos qué ocurrió en fechas subsecuentes, pues no se registraron muestreos de nuevo hasta julio de 2015. De acuerdo con la guía de la OMS, el 12 de agosto y el 24 de septiembre de 2015 todavía hay casos fuera del límite. Resalta de la Figura 1, que el 21 de octubre de 2014, 13 sitios excedían el límite; todavía resulta más significativo si agregamos el hecho de que ese día fueron muestreados únicamente 25 del total de los 34 sitios.

Por otra parte, en la Figura 2 se muestra la distribución espacial del arsénico según el número de veces que se excedió el límite permisible de la norma mexicana y la guía de la OMS por sitio de muestreo. 
Amenazas para la salud en el Río Sonora: análisis exploratorio de la calidad del agua reportada en la base de datos oficial de México

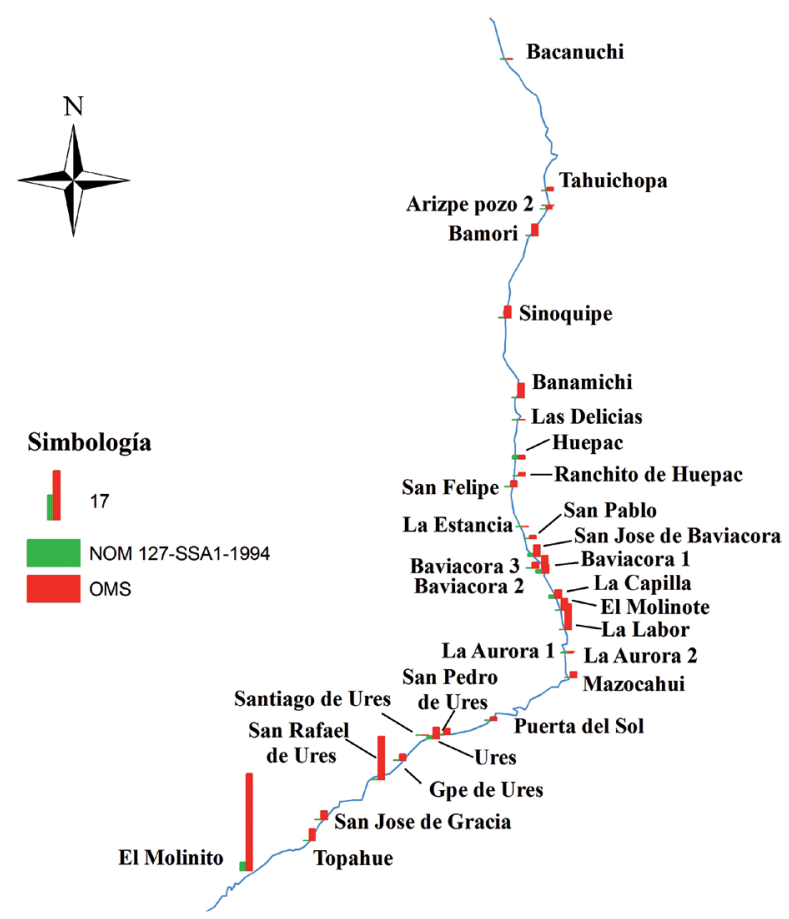

Figura 2. Número de registros de arsénico fuera del límite de la NOM 127-SSA1-1994 y de la OMS según los sitios de muestreo. Fuente: Elaboración propia con base en página oficial del Fideicomiso Río Sonora ${ }^{3}$

Como se observa en la Figura 2, de acuerdo con la norma mexicana, el sitio donde se presentaban más casos es El Molinito, con tres; mientras que según la guía de la OMS, los lugares donde se presentaban más casos son La Labor, San Rafael de Ures y El Molinito. En La Labor se hicieron 30 muestreos a lo largo del período y nueve de ellos excedían el límite de la Guía de la OMS. En San Rafael de Ures se hicieron 21 muestreos, de los cuales 15 rebasaban el valor recomendado en la guía. En el caso de El Molinito, pozo ubicado en la localidad Molino de Camou, de los 46 muestreos realizados a lo largo del período reportado en la base de datos, se excedió 34 veces la guía de la OMS. De hecho, los dos casos que todavía sobrepasaban la guía de la OMS en 2015 (ver Figura 1), son del pozo ubicado en el Molino de Camou: 12 de agosto y 24 de septiembre de 2015.

Estos resultados indican que el arsénico se encuentra en concentraciones más altas en la parte baja de la cuenca, contiguo a las localidades aledañas a la presa El Molinito, muy cerca de la ciudad de Hermosillo. El hecho de que solamente nueve casos excedían el límite permisible de arsénico establecido en la norma mexicana puede dar una falsa idea de seguridad en el agua de consumo, pues se ha encontrado que incluso a concentraciones menores a $0.025 \mathrm{mg} / \mathrm{L}$ es posible observar daño en la salud humana ${ }^{10}$. Si seguimos el criterio recomendado para arsénico en la guía de la OMS, el número de concentraciones fuera del límite aumenta hasta llegar a un total de 120 , lo cual evidencia el riesgo de padecer daños a la salud en la población expuesta, sobre todo si consideramos que este riesgo es exacerbado por la vulnerabilidad de las localidades que no cuentan con otra fuente alterna de suministro de agua potable ni con planta potabilizadora ${ }^{11}$.

Esta primera aproximación a la base de datos oficial permitió detectar no sólo algunas tendencias en el comportamiento espacio-temporal de algunos metales y parámetros fisicoquímicos de la calidad del agua, sino que también demuestra la falta de consistencia en la captura de datos del monitoreo que se supone implementado desde que ocurrió el derrame. Por un período de tres meses, de julio a septiembre de 2015, no se observan valores numéricos para la mayoría de los metales estudiados, sino solamente la abreviatura "NE", cuyo significado no se especifica; durante seis meses, de enero a junio de 2015 , no hay registros de muestreo alguno; además que la frecuencia de captura varía para ciertos parámetros en días, semanas y hasta meses, como es el caso de sulfatos, turbiedad, sólidos disueltos totales, conductividad electrolítica, $\mathrm{pH}$ y temperatura.

La discrepancia entre los límites permisibles de la NOM 127-SSA1-1994 y el valor recomendado en las guías de la OMS respecto al arsénico, resulta una preocupación adicional a las deficiencias encontradas en la base de datos. Las diferentes fuentes de exposición a pequeñas cantidades de arsénico, tanto naturales como laborales ${ }^{12}$, han motivado que desde 2006 organismos internacionales fijen sus recomendaciones en niveles lo más bajo posible y que al mismo tiempo sean tecnológicamente alcanzables ${ }^{13}$.

El derrame de lixiviados tóxicos al Río Sonora aumenta la lista de eventos desafortunados de alto impacto para el ambiente y la salud provocados por actividades mineras. Accidentes como el sucedido en España en 1998, donde se derramaron más de 5 millones de metros cúbicos de agua acidificada y lodos que contenían miles de toneladas de metales y arsénico, afectando un área natural protegida de más de 50,000 hectáreas de superficie, al parecer no son suficientes para aprender la lección ${ }^{14,15}$. Es necesario tomar conciencia de que el desbalance jurídicoinstitucional a favor de una minería a cielo abierto, industrial y masiva, no contribuirá a un verdadero desarrollo sostenible, sino más bien irá en perjuicio de la salud de la población actualmente expuesta, condenando el destino de futuras generaciones ${ }^{16}$. 


\section{AGRADECIMIENTOS}

Los autores agradecen el apoyo otorgado para la realización de este estudio por el Instituto Interamericano para la Investigación del Cambio Global (IAI) CRN3056, que a su vez es apoyado por la Fundación Nacional de Ciencias de los Estados Unidos (Grant GEO-1128040). Asimismo agradecen el apoyo y el compañerismo de los integrantes de la Red Fronteriza de Salud y Ambiente A.C.

\section{REFERENCIAS}

1. Aburto O, Rojo J, Ezcurra E. Los impactos de la minería de oro: repensando una minería sustentable. La Jornada Ecológica. 2015; 200: 4-5.

2. Lambertz M, Dergam JA. Mining disaster: Huge species impact. Nature. 2015; 528(7580): 39. DOI: 10.1038/528039b.

3. SEMARNAT. Derrame de sulfato de cobre en el Río Bacanuchi (afluente del Río Sonora). Secretaría del Medio Ambiente y Recursos Naturales; 2014.

4. Enciso A. Semarnat: desastre ambiental en Sonora, el peor de la minería en el país. La Jornada [Internet]. 26 Ago 2014.

5. SEMARNAT. Fideicomiso Río Sonora [Internet]. Secretaría del Medio Ambiente y Recursos Naturales; 2015.

6. DOF. Modificación a la Norma Oficial Mexicana NOM-127-SSA1-1994, Salud ambiental. Agua para uso y consumo humano. Límites permisibles de calidad y tratamientos a que debe someterse el agua para su potabilización. Diario Oficial de la Federación. México; 22 Nov 2000.
7. WHO. Guidelines for Drinking-water Quality. 4a ed. Switzerland: World Health Organisation; 2011.

8. Baba A, Tayfur G. Groundwater contamination and its effect on health in Turkey. Environ Monit Assess. 2011; 183(1-4): 77-94.

9. Rahman MM, Dong Z, Naidu R. Concentrations of arsenic and other elements in groundwater of Bangladesh and West Bengal, India: potential cancer risk. Chemosphere. 2015; 139: 54-64. DOI: 0.1016/j.chemosphere.2015.05.051.

10. Chakraborti D, Rahman MM, Mukherjee A, Alauddin M, Hassan M, Dutta RN, etal. Groundwater arsenic contamination in Bangladesh-21 Years of research. J Trace Elem Med Biol. 2015; 31:237-48. DOI: 10.1016/j.jtemb.2015.01.003.

11. Díaz-Caravantes RE. Balancing urban and peri-urban exchange: water geography of rural livelihoods in Mexico. Geogr J. 2012; 78(1): 42-53.

12. Joseph T, Dubey B, McBean EA. A critical review of arsenic exposures for Bangladeshi adults. Sci Total Environ. 2015; 527-528: 540-551. DOI: 10.1016/j. scitotenv.2015.05.035.

13. WHO. Guidelines for Drinking-water Quality. 3a ed. Switzerland: World Health Organisation; 2006.

14. Grimalt JO, Ferrer M, Macpherson E. The mine tailing accident in Aznalcollar. Sci Total Environ. 1999; 242(1-3): 3-11. DOI: 10.1016/S00489697(99)00372-1.

15. Moreno Millán E, Valdés Morillo F. Intervenciones de emergencia en Salud Pública tras el vertido tóxico por el accidente minero de Aznalcóllar (Sevilla). Emergencias. 2003;15:157-164.

16. Narchi NE, Búrquez Montijo A, Wider BT. Introducción. La Jornada Ecológica. 2015; 200: 2-3. 